\title{
OTIMIZAÇÃO E VALIDAÇÃo DE MÉTODOS ANALÍTICOS PARA DETERMINAÇÃO DE BTEX EM ÁGUA UTILIZANDO EXTRAÇÃO POR HEADSPACE E MICROEXTRAÇÃO EM FASE SÓLIDA
}

Fernanda F. Heleno, Aniel C. Lima, Robson J. C. F. Afonso e Mauricio X. Coutrim*

Departamento de Química, Universidade Federal de Ouro Preto, Campus Morro do Cruzeiro, s/n, 35400-000 Ouro Preto - MG, Brasil

Recebido em 2/3/09; aceito em 18/8/09; publicado na web em 21/1/10

EVALUATION OF ANALYTICAL METHODS FOR BTEX ANALYSIS IN WATER USING EXTRACTION BY HEADSPACE (HS) AND SOLID PHASE MICROEXTRACTION (SPME). Three analytical methods for the determination of BTEX in water were optimized and validated. With the best method the analytes were extracted of $10 \mathrm{~mL}$ of sample with $2.50 \mathrm{~g}$ of $\mathrm{NaCl}$ in headspace vial of $20 \mathrm{~mL}$ by $\mathrm{HS}$ and $\mathrm{SPME}$ to $40^{\circ} \mathrm{C}$ for $30 \mathrm{~min}$ for adsorption and to $250^{\circ} \mathrm{C}$ for $4 \mathrm{~min}$ for desorption and were analyzed by GCMS. The recovery was between $97.9 \%$ and $104.3 \%$, and the limit of detection was $2.4 \mathrm{ng} \mathrm{L}^{-1}$ for o-xylene. This method was using to analyze BTEX in water supply and surface water in Ouro Preto city. No sample had concentrations of BTEX above the legislation.

Keywords: SPME; headspace; BTEX.

\section{INTRODUÇÃOO}

Os compostos aromáticos, especialmente os hidrocarbonetos monoaromáticos denominados BTEX (benzeno, tolueno, etilbenzeno e os isômeros de xileno), estão entre os principais contaminantes das águas.

A presença destes compostos está comumente associada a deposições atmosféricas, vazamentos de petróleo e alguns de seus derivados, efluentes químicos industriais, dentre outros, devido à alta toxicidade, representa um risco à saúde humana e animal. A exposição humana a estes compostos pode levar ao desenvolvimento de problemas de saúde, desde irritação de olhos, mucosas e pele, passando por enfraquecimento do sistema nervoso central, depressão da medula óssea, até o desenvolvimento de câncer, no caso do benzeno, um composto classificado pela Organização Mundial de Saúde como potente agente carcinogênico. ${ }^{1}$

No Brasil, o Ministério da Saúde, através da Portaria 518/04, determina que os valores máximos permitidos (VMP) para benzeno, tolueno, etilbenzeno e xilenos em água para consumo humano são de 5, 170, 200 e $300 \mu \mathrm{g} \mathrm{L}^{-1}$, respectivamente. ${ }^{2}$

Devido à necessidade de se analisar esses compostos em baixas concentrações, os mesmos são isolados da matriz ambiental e analisados por cromatografia a gás (CG) e detecção por ionização em chama (DIC) ou espectrometria de massas (EM), porém, os métodos analíticos mais sensíveis recorrem a processos de pré-concentração. É muito raro se encontrar uma pesquisa publicada para a determinação de BTEX em água com injeção direta da amostra por causa dos baixos limites de detecção exigidos da análise. ${ }^{3}$ Dessa forma, muitas técnicas de extração têm sido empregadas para aumentar o limite de detecção de BTEX em água e dentre elas destacam-se a utilização de headspace estático e dinâmico, ${ }^{4-8}$ de purga e trapeamento, ${ }^{4,9-13}$ e de microextração em fase sólida com imersão direta da fibra na amostra, ${ }^{14}$ ou com os analitos extraídos na fase headspace..$^{5,15-23}$ Novos métodos de extração e concentração de BTEX e outros compostos orgânicos voláteis visando simplicidade e diminuição dos limites de detecção têm sido propostos. ${ }^{11,24-27}$

A extração de BTEX da amostra por headspace (HS), acoplado ou não a um sistema de microextração em fase sólida (MEFS), seguida pela análise com cromatografia a gás, é o modo mais utilizado para avaliação desses compostos em água..$^{4-8,14-23}$ Isso se dá principalmente devido aos baixos limites de detecção obtidos com essas técnicas, da simplicidade do ponto de vista instrumental, além de que os pro-

*e-mail: mcoutrim@iceb.ufop.br cedimentos dispensam o uso de solventes extratores e manipulação excessiva de amostra. A eficiência dos métodos de extração por HS ou MEFS sofre a influência de alguns parâmetros experimentais, tais como salinidade, temperatura, tempo de extração e proporção entre as fases. Com relação à salinidade, o efeito da força iônica pode aumentar ou diminuir as quantidades extraídas, dependendo do composto e da concentração do sal. Em geral a força iônica reduz ou aumenta a solubilidade do analito na matriz, de acordo com a polaridade do mesmo. ${ }^{28}$ É recomendado o uso de altas temperaturas em HS devido à melhor transferência de massa. Em MEFS, a influência da temperatura na partição do analito entre a fase estacionária e a amostra pode ser descrita em termos cinéticos e termodinâmicos. Teoricamente a quantidade extraída aumenta com a temperatura, devido à melhor transferência de massa; contudo, para um processo exotérmico o aumento de temperatura diminui o valor do coeficiente de partição entre a fibra e o analito. Portanto, diminuindo-se a temperatura aumenta-se a quantidade de analito extraída. Existe assim uma quantidade máxima extraída que depende da temperatura e das características do sistema de extração (amostra/fibra). ${ }^{29} \mathrm{O}$ tempo ótimo para a extração é definido como o tempo necessário para que o equilíbrio entre as fases seja estabelecido. A quantidade máxima extraída por MEFS, por se tratar de um processo de partição, é obtida quando o equilíbrio entre o analito e a fase estacionária é estabelecido. Para compostos que possuem coeficiente de partição menor, o tempo necessário para estabelecer o equilíbrio é longo e, nesse caso, o tempo ótimo é definido a partir da otimização de vários parâmetros. ${ }^{29}$ A relação entre o volume de amostra e o volume de headspace é um parâmetro importante que afeta a eficiência da extração.

Planejamentos fatoriais são extremamente úteis para medir os efeitos (ou influências) de uma ou mais variáveis na resposta de um processo. O usual é realizar um planejamento com dois níveis, os quais são identificados como nível superior (+) e inferior (-). Em geral, para $n$ níveis do fator $k$, o planejamento torna-se um fatorial $n^{k}$, onde o número mínimo de experimentos corresponde ao resultado desse fatorial. ${ }^{30}$ Planejamento experimental multivariado tem sido utilizado para a otimização das condições experimentais de extração de BTEX de água. ${ }^{19}$

Para que um sistema funcione adequadamente dentro das condições de análise para as quais foi desenvolvido é necessário que seja validado. Os parâmetros que necessitam ser calculados durante o processo de validação podem variar de acordo com o tipo de ensaio. ${ }^{31}$ Os parâmetros analíticos normalmente encontrados para validação 
de métodos cromatográficos são: seletividade, linearidade e faixa de aplicação, precisão, exatidão, limite de detecção, limite de quantificação e robustez. ${ }^{32}$

Três métodos para análise de BTEX em água utilizando extração por headspace e microextração em fase sólida do headspace seguidas de cromatografia a gás com detecção por ionização em chama (CG-DIC) e espectrometria de massas (CG-EM) foram avaliados. Os métodos foram validados nas condições otimizadas pelo planejamento experimental multivariado. BTEX em amostras ambientais de águas superficiais e de abastecimento coletadas em Ouro Preto, MG, foram extraídos por MEFS do HS e analisados por CG-EM, um dos métodos avaliados.

\section{PARTE EXPERIMENTAL}

\section{Reagentes}

Os reagentes benzeno, 99,7\%, benzoato de etila, orto-, meta- e para-xileno e etilbenzeno, todos $99 \%$, foram obtidos da SigmaAldrich $^{\circledR}$; o cloreto de sódio, 99\%, e metanol, 99,8\%, da J.T.Baker ${ }^{\circledR}$; e o tolueno, 99,5\%, da Vetec Química Fina Ltda.

As soluções contendo os padrões de BTEX foram preparadas todos os dias, em água ultrapura, Milli- ${ }^{\circledR}$, a partir da diluição de uma solução metanólica estoque padrão, contendo benzeno, tolueno, orto-, meta-, para-xilenos e etilbenzeno nas concentrações de 880,0; 920,0; 140,0; 900,0; 660,0 e 230,0 $\mathrm{mg} \mathrm{L}^{-1}$, respectivamente. Essa solução foi armazenada sob refrigeração a $4{ }^{\circ} \mathrm{C}$ por um período não superior a 30 dias. A solução aquosa de benzoato de etila (padrão interno) na concentração de $20,0 \mathrm{mg} \mathrm{L}^{-1}$ foi preparada a partir de uma diluição de cem vezes da solução estoque desse padrão em metanol.

Em todas as determinações de BTEX, tanto nas otimizações quanto nas amostras coletadas, um branco com água Milli Q foi analisado nas mesmas condições analíticas, não sendo encontradas concentrações de BTEX acima do limite de detecção nesses brancos.

\section{Amostragem}

Amostras de água foram coletadas em triplicatas em 8 locais na

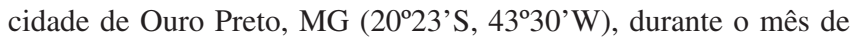
janeiro de 2009. Amostras de água superficiais foram coletadas em 3 locais no Ribeirão Tripuí, que atravessa uma fábrica de alumínio e recebe parte do esgoto da cidade. Foram coletadas amostras do ribeirão antes dele atravessar a fábrica de alumínio ( $\mathrm{S} 1)$, antes de receber esgoto (S2) e após esse recebimento (S3). Amostras de água também foram coletadas em 2 poços artesianos (P1 e P2) situados na parte mais baixa da cidade (1500 $\mathrm{m}$ do nível do mar) e distantes não mais que $50 \mathrm{~m}$ um do outro. Também foram coletadas amostras em 3 reservatórios de água potável que abastecem a Universidade Federal de Ouro Preto (UFOP). Um dos reservatórios é subterrâneo e situa-se a cerca de $100 \mathrm{~m}$ dos poços artesianos (R1) e os outros dois estão situados no campus da UFOP, na parte alta da cidade (1650 $\mathrm{m}$ do nível do mar), sendo um subterrâneo (R2) e o outro elevado a 5 m do nível do solo (R3). Um posto de gasolina desativado há mais de 3 anos situava-se a cerca de $300 \mathrm{~m}$ dos poços artesianos e do reservatório R1.

Frascos headspace em vidro borossilicato de $20 \mathrm{~mL}$ e pipeta volumétrica de $10 \mathrm{~mL}$ depois de lavados foram deixados em uma solução aquosa de ácido nítrico, 2,5\%, m/v, por $24 \mathrm{~h}$. Em seguida, foram rinsados com água Milli- ${ }^{\circledR}$, secos e isolados adequadamente até a amostragem. Antes da amostragem foram adicionados 2,50 g de cloreto de sódio em cada frasco headspace. Para a amostragem de água dos poços artesianos, dos reservatórios e do Ribeirão Tripuí, um volume de $10,00 \mathrm{~mL}$ de amostra foi coletado diretamente para frascos de $20 \mathrm{~mL}$ de capacidade contendo o sal. Em seguida, foram adicionados $100 \mu \mathrm{L}$ de uma solução aquosa de benzoato de etila (padrão interno) na concentração de $20,0 \mathrm{mg} \mathrm{L}^{-1}$ em cada frasco e os mesmos foram lacrados com tampas de alumínio e septo de silicone/teflon. Após a coleta os frascos foram armazenados a cerca de $5{ }^{\circ} \mathrm{C}$ em recipientes apropriados até a chegada ao laboratório, onde foram transferidos para um refrigerador e permaneceram aí a $4{ }^{\circ} \mathrm{C}$ até análise.

\section{Determinação por headspace estático}

Para a determinação de BTEX por headspace, $10,00 \mathrm{~mL}$ da solução a ser analisada foram acondicionados em frascos de vidro de $20 \mathrm{~mL}$ contendo $2,50 \mathrm{~g}$ de cloreto de sódio e $100 \mu \mathrm{L}$ de benzoato de etila na concentração de $20,0 \mathrm{mg} / \mathrm{L}$. Em seguida, o frasco, próprio para headspace, foi lacrado com tampa de alumínio contendo septo de silicone faceado com teflon e agitado vigorosamente por $1 \mathrm{~min}$. Após esse período, o frasco foi mantido envolto em um bloco de alumínio aquecido a $80{ }^{\circ} \mathrm{C}$ por $30 \mathrm{~min}$. Por meio de uma seringa gastight, 1,00 mL da fase vapor (headspace) foi recolhido à meia altura entre a tampa do frasco e a superfície do líquido e injetado no cromatógrafo. A seringa era previamente lavada com metanol e aquecida a $80^{\circ} \mathrm{C}$ por $5 \mathrm{~min}$. A fase vapor era imediatamente amostrada e injetada com a seringa ainda aquecida para evitar condensação do vapor no seu interior.

\section{Extração por MEFS no headspace}

Um volume de 10,00 mL de solução contendo BTEX foi acondicionado em frascos de $20 \mathrm{~mL}$ contendo 2,50 g de cloreto de sódio e $100 \mu \mathrm{L}$ de benzoato de etila na concentração de 20,0 mg/L. Em seguida, o frasco foi vedado e agitado vigorosamente por $1 \mathrm{~min}$. Inseriu-se a agulha do dispositivo de MEFS no frasco, expondo a fibra na fase headspace. Utilizou-se a fibra SPME Carboxen/Polydimethysiloxane, 24 ga, $75 \mu \mathrm{m}$ (Supelco ${ }^{\mathrm{TM}}$ ), previamente condicionada a $300{ }^{\circ} \mathrm{C}$ por $60 \mathrm{~min}$. $\mathrm{O}$ frasco com a fibra foi mantido envolto em um bloco de alumínio, aquecido a $40{ }^{\circ} \mathrm{C}$, por $15 \mathrm{~min}$. Após o tempo de extração, a fibra foi reposicionada dentro da agulha e o dispositivo foi imediatamente levado ao injetor do cromatógrafo para dessorção dos analitos, sendo aí deixada por $4 \min$ a $250{ }^{\circ} \mathrm{C}$.

\section{Análises cromatográficas}

As melhores condições cromatográficas foram obtidas visando a melhor resolução dos picos dos analitos no cromatograma com base em testes univariados. As análises por cromatografia gasosa com detecção por ionização em chama (CG-DIC) foram realizadas em um cromatógrafo a gás, Varian, modelo CP-3380, utilizando-se uma coluna Rtx ${ }^{\circledR}$-5MS (5\% fenil, 95\% dimetilpolissiloxano), da Restek ${ }^{\circledR}$ (30 m x 0,25 mm x 0,25 $\mu \mathrm{m}$ ). O injetor foi mantido a $250{ }^{\circ} \mathrm{C}$, sem divisão de fluxo (modo splitless) por 0,5 min e, após esse tempo, com divisão de fluxo (split) na razão de 1:30. Durante as análises o forno da coluna foi mantido a $35^{\circ} \mathrm{C}$ por $10 \mathrm{~min}$, depois a $225^{\circ} \mathrm{C}$ por $1 \mathrm{~min}$, com rampa de aquecimento de $20^{\circ} \mathrm{C} \mathrm{min}-1$. O detector foi mantido a $280{ }^{\circ} \mathrm{C}$. O volume injetado de amostra foi de $1000 \mu \mathrm{L}$. Foi empregado hidrogênio como gás de arraste a uma vazão de 1,8 $\mathrm{mL}$ minn $^{-1}$ e nitrogênio como gás make-up, com a chegada de ambos ao detector a uma vazão de $30 \mathrm{~mL} \mathrm{~min}{ }^{-1}$. A chama do detector foi mantida com ar sintético a $300 \mathrm{~mL} \mathrm{~min}^{-1}$ e hidrogênio a $30 \mathrm{~mL} \mathrm{~min}$ ${ }^{1}$. Os cromatogramas foram obtidos e tratados pelo software Varian Star Workstation ${ }^{\circledR}$.

Para as análises por cromatografia gasosa com detecção por espectrometria de massas (CG-EM) utilizou-se um equipamento GC-MS Shimadzu, modelo GC-2010, com uma coluna Rtx ${ }^{\circledR}-5$ MS, 
(5\% fenil, 95\% dimetilpolissiloxano), da $\operatorname{Restek}^{\circledR}$ (30 m x 0,25 mm x $0,25 \mu \mathrm{m})$. Utilizou-se um liner com diâmetro interno de $0,75 \mathrm{~mm}$, especial para MEFS. O injetor foi mantido a $200{ }^{\circ} \mathrm{C}$ para o procedimento utilizando somente HS e $250{ }^{\circ} \mathrm{C}$ para HS e MEFS, com divisão de fluxo (split) na razão de 1:15. A temperatura do forno da coluna foi mantida a $40{ }^{\circ} \mathrm{C}$ por $1 \mathrm{~min}$, em seguida foi elevada a 70 ${ }^{\circ} \mathrm{C}$, com taxa de aquecimento de $5^{\circ} \mathrm{C} \min ^{-1} \mathrm{e}$, finalmente, foi a 220 ${ }^{\circ} \mathrm{C}$, com alta taxa de aquecimento de $60{ }^{\circ} \mathrm{C} \mathrm{min}^{-1}$, e mantida assim por $0,5 \mathrm{~min}$. Hélio foi utilizado como gás de arraste com velocidade linear de $50,9 \mathrm{~cm} \mathrm{~s}^{-1}$. Um volume de $1000 \mu \mathrm{L}$ de amostra contida no headspace foi injetado no cromatógrafo com uma seringa gastight.

Para a detecção por espectrometria de massas utilizou-se um detector contendo uma fonte de ionização por elétrons (EI-70 eV) e um analisador de massas quadrupolo, operado no modo varredura $(S C A N)$ para identificação e íons selecionados (SIM - selected ion monitoring) para quantificação dos compostos. Foram monitorados o íon principal do benzeno com razão $\mathrm{m} / \mathrm{z} 78$ e o íon principal dos demais compostos com razão $\mathrm{m} / \mathrm{z}$, 91. Também foram monitorados os íons secundários com $\mathrm{m} / \mathrm{z}, 77$ e 52 do benzeno e $\mathrm{m} / \mathrm{z}, 92$ e 65 do tolueno. Para os demais compostos foram monitorados os íons secundários com $\mathrm{m} / \mathrm{z}, 106 \mathrm{e} 77$. A interface foi mantida a $250{ }^{\circ} \mathrm{C}$ e a fonte de íons a $200{ }^{\circ} \mathrm{C}$.

\section{Planejamento fatorial}

As condições analíticas para extração de BTEX de água por headspace estático foram otimizadas pelo planejamento fatorial completo com quatro fatores e triplicata no ponto central $\left(2^{4-0}\right)$. Os parâmetros otimizados foram: massa de cloreto de sódio utilizada na amostra devido ao efeito salting out, volume de amostra dentro do frasco headspace, tempo e temperatura de extração. Foram realizados 19 experimentos. Para a extração de BTEX por microextração em fase sólida do headspace utilizou-se o planejamento fatorial fracionário $\left(2^{6-1}\right)$ com determinação do ponto central em triplicata. Os parâmetros otimizados foram: massa de cloreto de sódio, tempos e temperaturas de adsorção de BTEX à fibra e de dessorção da mesma, e volume de amostra dentro do frasco headspace. Foram realizados 35 experimentos.

\section{Validação}

A seletividade, a linearidade, a detectabilidade, a precisão, a exatidão e a robustez foram determinadas para validar os métodos avaliados. A seletividade foi determinada através da análise de uma matriz de água superficial pouco contaminada do Ribeirão Tripuí, onde a presença dos analitos foi verificada por CG-EM. A linearidade foi determinada pelos coeficientes de correlação das curvas analíticas construídas com seis diferentes concentrações de cada BTEX adicionadas a uma matriz pouco contaminada do Ribeirão Tripuí para o método com detecção por ionização em chama. Para os outros dois métodos utilizaram-se sete concentrações para a construção da curva analítica. A detectabilidade foi avaliada pela determinação dos limites de detecção e quantificação pelo método da relação sinal/ruído dos compostos nos cromatogramas das soluções com as menores concentrações de BTEX utilizadas nas curvas analíticas. A precisão foi determinada pelas variâncias nas concentrações obtidas da análise em sete replicatas de uma solução padrão de BTEX. Para a detecção pelo DIC utilizou-se uma solução com concentração de $100 \mu \mathrm{g} \mathrm{L} \mathrm{L}^{-1} \mathrm{e}$, para os métodos com detecção por espectrometria de massas, de $50 \mu \mathrm{g} \mathrm{L}^{-1}$. A exatidão foi determinada pela recuperação das concentrações de BTEX adicionadas em amostras coletadas no Ribeirão Tripuí. Fizeram-se adições de BTEX em três concentrações diferentes relativas a valores próximos ao menor, maior e central da curva analítica e, para cada nível de adição, as determinações foram realizadas em nove replicatas. Para a escolha dos parâmetros a serem avaliados para verificação da robustez utilizaram-se os resultados do planejamento experimental multivariado realizado anteriormente. Para o método por headspace foram avaliados os efeitos nas variações da quantidade de cloreto de sódio (2,00-2,50 g) e da temperatura $\left(70-80{ }^{\circ} \mathrm{C}\right)$ e para o método de MEFS foram avaliados os efeitos nas variações da quantidade de cloreto de sódio $(2,00-2,50 \mathrm{~g})$ e da temperatura de adsorção à fibra $\left(30-40{ }^{\circ} \mathrm{C}\right)$.

\section{RESULTADOS E DISCUSSÃO}

Na otimização das condições analíticas dos métodos para avaliação de compostos BTEX em água foram escolhidos os três métodos analíticos mais comumente utilizados para esse fim: extração dos analitos por headspace com determinação por CG-DIC e por CGEM e extração destes por headspace e MFES com determinação por CG-EM. Devido aos diversos parâmetros analíticos a serem otimizados simultaneamente, tais como, tempo e temperatura de extração, quantidade de sal na solução, relação entre as fases líquida e vapor, além do controle de temperatura e tempo para a extração por MEFS, optou-se pela otimização das condições utilizando a técnica de planejamento experimental multivariado. ${ }^{5}$ Antes da realização dos experimentos envolvendo as extrações definidas pelo planejamento fatorial, as condições cromatográficas foram otimizadas visando a melhor resolução dos analitos no menor tempo, sem interferências de outros compostos presentes nas matrizes avaliadas. Em nenhuma condição foi possível obter os isômeros $m$, $p$-xilenos separados no cromatograma. Na Figura 1 é apresentado o cromatograma de uma amostra de água do Ribeirão Tripuí coletada antes do mesmo receber carga de poluentes, a qual foi contaminada com BTEX na concentração de $100 \mu \mathrm{g} \mathrm{L}^{-1}$ e, após extração por HS, foi analisada por CG-DIC.

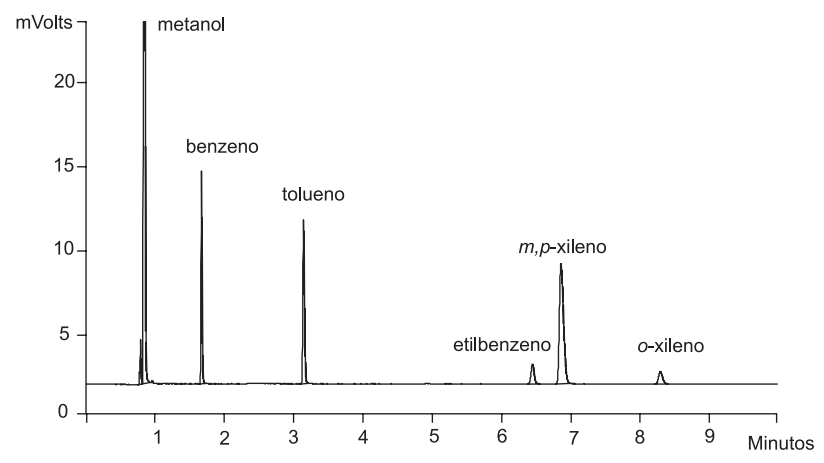

Figura 1. Cromatograma do extrato de HS de uma amostra de água do Ribeirão Tripuí contaminada com $100 \mu \mathrm{g} L^{-1}$ de BTEX analisada por CG-DIC

\section{Otimização dos parâmetros de extração}

Utilizou-se o planejamento fatorial completo para a otimização das condições analíticas do método de extração de BTEX da água com headspace e determinação dos mesmos por CG-DIC e CG-EM. Os experimentos relativos ao ponto central foram realizados em triplicata e os valores das variáveis ensaiadas foram definidos com base em estudos semelhantes. ${ }^{5,7,8,15-17,22}$ A matriz dos experimentos e os vetores de resposta (áreas dos picos cromatográficos) obtidos pelo planejamento fatorial do tipo $2^{4}$ (quatro fatores em dois níveis) são apresentados na Tabela 1 .

Para o método que utilizou a extração dos analitos por HS e MEFS e determinação por CG-EM, devido ao número elevado de parâmetros a serem otimizados, realizou-se um planejamento fatorial fracionário do tipo $2^{6-1}$ para verificar a significância dos fatores dentro dos seus respectivos domínios, incluindo ponto central em triplicata. 
Tabela 1. Matriz para o planejamento fatorial com quatro fatores em dois níveis $\left(2^{4-0}\right)$ e as áreas dos picos dos cromatogramas obtidos para os métodos de extração de BTEX por HS e análise com CG-DIC e com CG-EM

\begin{tabular}{|c|c|c|c|c|c|c|c|c|c|}
\hline \multirow[b]{2}{*}{ Ensaio } & \multicolumn{4}{|c|}{ Fatores } & \multicolumn{5}{|c|}{ Área dos picos cromatográficos } \\
\hline & $\begin{array}{l}\text { Sal } \\
(\mathrm{g})\end{array}$ & $\begin{array}{l}\text { Tempo } \\
(\mathrm{min})\end{array}$ & $\begin{array}{c}\text { Temperatura } \\
\left({ }^{\circ} \mathrm{C}\right)\end{array}$ & $\begin{array}{c}\text { Fração de fase } \\
(\%)\end{array}$ & Benzeno & Tolueno & Etilbenzeno & $m, p$-xilenos & $o$-xilenc \\
\hline 1 & 0,00 & 10 & 40 & 50 & 2095 & 1601 & 266 & 1961 & 160 \\
\hline 2 & 2,50 & 10 & 40 & 50 & 3490 & 2614 & 364 & 3543 & 299 \\
\hline 3 & 0,00 & 30 & 40 & 50 & 4791 & 4854 & 1039 & 8163 & 855 \\
\hline 4 & 2,50 & 30 & 40 & 50 & 12147 & 13857 & 3140 & 25524 & 2498 \\
\hline 5 & 0,00 & 10 & 80 & 50 & 7611 & --- & --- & --- & --- \\
\hline 6 & 2,50 & 10 & 80 & 50 & 13955 & 15818 & 3338 & 6634 & 2878 \\
\hline 7 & 0,00 & 30 & 80 & 50 & 3598 & 3335 & 629 & 5000 & 392 \\
\hline 8 & 2,50 & 30 & 80 & 50 & 6161 & 5095 & 788 & 6696 & 462 \\
\hline 9 & 0,00 & 10 & 40 & 75 & 1722 & 1106 & 126 & 1025 & 0 \\
\hline 10 & 2,50 & 10 & 40 & 75 & 7778 & 7592 & 1478 & 12380 & 1005 \\
\hline 11 & 0,00 & 30 & 40 & 75 & 3558 & 3187 & 559 & 4357 & 380 \\
\hline 12 & 2,50 & 30 & 40 & 75 & 23409 & 28228 & 6466 & 53208 & 4899 \\
\hline 13 & 0,00 & 10 & 80 & 75 & 12623 & 13601 & 2758 & 22610 & 2182 \\
\hline 14 & 2,50 & 10 & 80 & 75 & 20314 & 24646 & 5755 & 48235 & 4464 \\
\hline 15 & 0,00 & 30 & 80 & 75 & 6879 & 7366 & 1560 & 12873 & 1048 \\
\hline 16 & 2,50 & 30 & 80 & 75 & 9564 & 11051 & 2603 & 21220 & 3402 \\
\hline $17(\mathrm{C})$ & 1,25 & 20 & 60 & 62,5 & 9353 & 10710 & 2460 & 19925 & 1926 \\
\hline $18(\mathrm{C})$ & 1,25 & 20 & 60 & 62,5 & 2095 & 1601 & 266 & 1961 & 160 \\
\hline 19(C) & 1,25 & 20 & 60 & 62,5 & 3490 & 2614 & 364 & 3543 & 299 \\
\hline
\end{tabular}

A matriz dos experimentos e os vetores de resposta (áreas dos picos cromatográficos) obtidos são apresentados na Tabela 2. Em ambas as situações, as soluções com concentração de BTEX em torno de $100 \mu \mathrm{g} \mathrm{L}^{-1}$ foram agitadas manualmente por $1 \mathrm{~min}$ antes da extração. Com os resultados obtidos, utilizando o programa Statistica 7.0 (StatSoft, Inc. USA, 2004) foram gerados os diagramas de pareto. Os gráficos de pareto do planejamento fatorial para os métodos de HS com CG-DIC e CG-EM indicaram que a influência do fator temperatura é muito superior aos demais fatores para todos os compostos analisados. O efeito do fator fração de fase foi negativo para todos os analitos com exceção dos isômeros $m$, $p$-xilenos, indicando que com uma relação de volume de água/headspace igual a 3:1 (nível alto desse fator, igual a 75\%) obtém-se um menor percentual de compostos extraídos. Um volume de amostra muito maior do que o espaço ocupado pela fase de vapor acima dele é desfavorável para a extração por headspace estático da maioria dos compostos avaliados por esses métodos. Esse comportamento pode ser observado no diagrama de pareto do etilbenzeno apresentado na Figura 2, que foi muito semelhante aos demais analitos. A condição que proporcionou a resposta mais satisfatória para esses dois métodos foi: temperatura de $80{ }^{\circ} \mathrm{C}$, tempo de extração de $30 \mathrm{~min}$, $2,50 \mathrm{~g}$ de cloreto de sódio e fração de fase de $50 \%$, ou seja, 10,0 $\mathrm{mL}$ de solução e $10,0 \mathrm{~mL}$ de headspace.

Nos diagramas de pareto do planejamento fatorial para o método HS e MEFS com CG-EM pode ser observado que o uso de cloreto de sódio é o fator mais significativo para os BTEX, com um efeito positivo sobre a extração, aumentando a quantidade extraída, como pode ser observado na Figura 3 para o etilbenzeno.

As temperaturas de adsorção e de dessorção apresentaram efeitos significativos para benzeno, tolueno e $o$-xileno. Para a temperatura de dessorção o efeito foi negativo e a temperatura de adsorção apresentou efeito positivo sobre a extração. Os tempos de adsorção e de dessorção apresentaram efeitos significativos positivos apenas para benzeno e tolueno. Nessa condição, o aumento do tempo de extração proporcionou maior rendimento na extração, resultado esse que pode ser corroborado com dados da

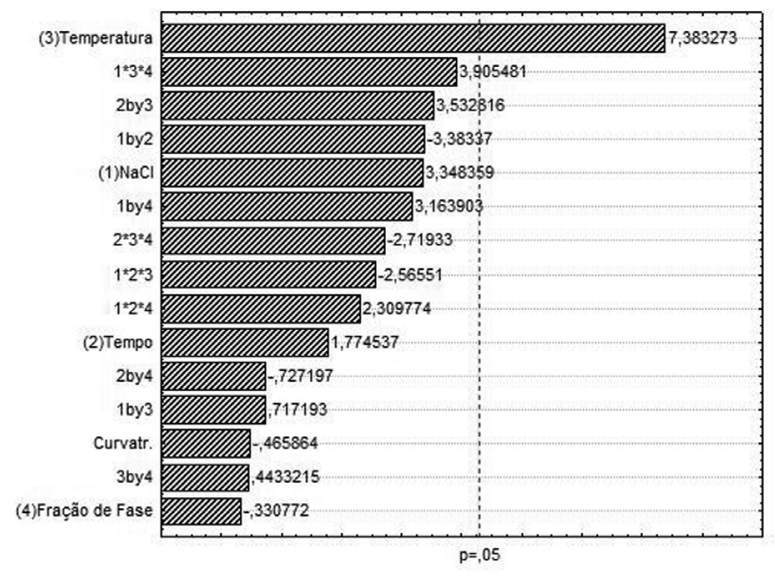

Figura 2. Diagrama de pareto do planejamento fatorial para etilbenzeno extraído por $H S$

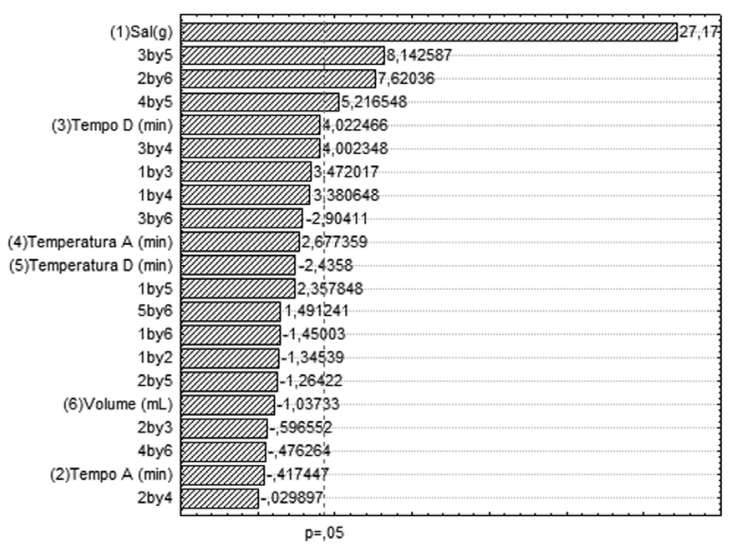

Figura 3. Diagrama de pareto do planejamento fatorial para etilbenzeno extraído por MEFS 
Tabela 2. Matriz para o planejamento fatorial fracionário com cinco fatores em dois níveis $\left(2^{6-1}\right)$ e as áreas dos picos dos cromatogramas obtidos para os métodos de extração de BTEX por HS e determinação por CG-EM

\begin{tabular}{|c|c|c|c|c|c|c|c|c|c|c|c|}
\hline \multirow[b]{2}{*}{ Ensaio } & \multicolumn{6}{|c|}{ Fatores } & \multicolumn{5}{|c|}{ Áreas dos picos cromatográficos } \\
\hline & $\begin{array}{l}\text { Sal } \\
(\mathrm{g}) \\
\end{array}$ & $\begin{array}{c}\text { Tempo * } \\
(\mathrm{min})\end{array}$ & $\begin{array}{c}\text { Tempo** } \\
(\mathrm{min}) \\
\end{array}$ & $\begin{array}{c}\text { Temp. }{ }^{*} \\
\left({ }^{\circ} \mathrm{C}\right) \\
\end{array}$ & $\begin{array}{c}\text { Temp. }{ }^{* *} \\
\left({ }^{\circ} \mathrm{C}\right) \\
\end{array}$ & $\begin{array}{c}\text { Fração de fase } \\
\begin{array}{c}(\%) \\
\end{array}\end{array}$ & Benzeno & Tolueno & Etilbenze-no & $m, p$-xilenos & $o$-xileno \\
\hline 1 & 0,00 & 10 & 20 & 26 & 250 & 50 & 63468 & 63051 & 11015 & 97593 & 7421 \\
\hline 2 & 2,50 & 10 & 20 & 26 & 250 & 75 & 134397 & 158260 & 33207 & 293618 & 23881 \\
\hline 3 & 0,00 & 30 & 20 & 26 & 250 & 75 & 80724 & 77705 & 13444 & 117590 & 9431 \\
\hline 4 & 2,50 & 30 & 20 & 26 & 250 & 50 & 201761 & 221915 & 41410 & 393420 & 50263 \\
\hline 5 & 0,00 & 10 & 40 & 26 & 250 & 75 & 62370 & 59722 & 10715 & 95545 & 7051 \\
\hline 6 & 2,50 & 10 & 40 & 26 & 250 & 50 & 182729 & 208031 & 39947 & 372790 & 33557 \\
\hline 7 & 0,00 & 30 & 40 & 26 & 250 & 50 & 91377 & 79418 & 12614 & 108497 & 9234 \\
\hline 8 & 2,50 & 30 & 40 & 26 & 250 & 75 & 145532 & 165302 & 32354 & 287777 & 20735 \\
\hline 9 & 0,00 & 10 & 20 & 40 & 250 & 75 & 73933 & 73066 & 13664 & 119277 & 8861 \\
\hline 10 & 2,50 & 10 & 20 & 40 & 250 & 50 & 177033 & 196703 & 37666 & 355606 & 38130 \\
\hline 11 & 0,00 & 30 & 20 & 40 & 250 & 50 & 98761 & 86239 & 13443 & 120733 & 10535 \\
\hline 12 & 2,50 & 30 & 20 & 40 & 250 & 75 & 169559 & 191022 & 36515 & 338619 & 29275 \\
\hline 13 & 0,00 & 10 & 40 & 40 & 250 & 50 & 72742 & 64724 & 10808 & 96075 & 8051 \\
\hline 14 & 2,50 & 10 & 40 & 40 & 250 & 75 & 108114 & 125554 & 25566 & 233634 & 17192 \\
\hline 15 & 0,00 & 30 & 40 & 40 & 250 & 75 & 81691 & 70028 & 11104 & 103262 & 9006 \\
\hline 16 & 2,50 & 30 & 40 & 40 & 250 & 50 & 140906 & 150545 & 27765 & 266490 & 28775 \\
\hline 17 & 0,00 & 10 & 20 & 26 & 280 & 75 & 48335 & 50218 & 10315 & 85154 & 6393 \\
\hline 18 & 2,50 & 10 & 20 & 26 & 280 & 50 & 127008 & 137228 & 26259 & 242799 & 20994 \\
\hline 19 & 0,00 & 30 & 20 & 26 & 280 & 50 & 36778 & 20676 & 2468 & 20308 & 2260 \\
\hline 20 & 2,50 & 30 & 20 & 26 & 280 & 75 & 107925 & 128302 & 29129 & 247227 & 19352 \\
\hline 21 & 0,00 & 10 & 40 & 26 & 280 & 50 & 54383 & 54354 & 11349 & 92230 & 7506 \\
\hline 22 & 2,50 & 10 & 40 & 26 & 280 & 75 & 91262 & 111605 & 25899 & 219714 & 16858 \\
\hline 23 & 0,00 & 30 & 40 & 26 & 280 & 75 & 54590 & 51446 & 10248 & 83986 & 7160 \\
\hline 24 & 2,50 & 30 & 40 & 26 & 280 & 50 & 113837 & 119424 & 24265 & 211103 & 19547 \\
\hline 25 & 0,00 & 10 & 20 & 40 & 280 & 50 & 77801 & 35726 & 5079 & 42763 & 4202 \\
\hline 26 & 2,50 & 10 & 20 & 40 & 280 & 75 & 91104 & 110372 & 24659 & 211740 & 16909 \\
\hline 27 & 0,00 & 30 & 20 & 40 & 280 & 50 & 73492 & 54114 & 8253 & 69069 & 6993 \\
\hline 28 & 2,50 & 30 & 20 & 40 & 280 & 50 & 105082 & 104303 & 18484 & 174873 & 15792 \\
\hline 29 & 0,00 & 10 & 40 & 40 & 280 & 75 & 31232 & 23226 & 3385 & 29560 & 2689 \\
\hline 30 & 2,50 & 10 & 40 & 40 & 280 & 50 & 296912 & 332188 & 67257 & 600066 & 62716 \\
\hline 31 & 0,00 & 30 & 40 & 40 & 280 & 50 & 136319 & 93522 & 11398 & 103862 & 10863 \\
\hline 32 & 2,50 & 30 & 40 & 40 & 280 & 75 & 211645 & 265669 & 57920 & 513118 & 42785 \\
\hline $33(\mathrm{C})$ & 1,25 & 20 & 30 & 33 & 265 & 62,50 & 186840 & 223935 & 46393 & 424341 & 31939 \\
\hline $34(\mathrm{C})$ & 1,25 & 20 & 30 & 33 & 265 & 62,50 & 180646 & 219432 & 44322 & 410330 & 29794 \\
\hline $35(\mathrm{C})$ & 1,25 & 20 & 30 & 33 & 265 & 62,50 & 174046 & 210817 & 41358 & 374500 & 27955 \\
\hline
\end{tabular}

* Adsorção. ** Dessorção

literatura, pois a MEFS se baseia na partição do analito entre duas fases requerendo, portanto, tempo necessário para que haja sorção do analito na fibra. ${ }^{29}$ Como observado com headspace, também com MEFS o efeito do fator fração de fase foi negativo para todos os BTEX, indicando que uma relação de volume água/headspace muito maior que 1 não favorece a adsorção desses na fibra. Deste modo, a condição que proporcionou a resposta mais satisfatória para o método HS e MEFS com CG-EM foi: temperatura de adsorção de $40{ }^{\circ} \mathrm{C}$, temperatura de dessorção de $250{ }^{\circ} \mathrm{C}$, tempo de adsorção de $30 \mathrm{~min}$, tempo de dessorção de $4 \mathrm{~min}, 2,50 \mathrm{~g}$ de cloreto de sódio e fração de fase de $50 \%$, ou seja, volume de 10,0 $\mathrm{mL}$ de amostra com 10,0 mL de headspace.

\section{Validação}

Para garantir que os três métodos fornecessem informações confiáveis e interpretáveis sobre a amostra, os métodos foram submetidos à validação. Foram determinados os limites de detecção e quantificação, a linearidade, a precisão, a seletividade, a robustez e a recuperação de cada método. Os métodos foram seletivos porque nos cromatogramas dos extratos obtidos, os espectros de massas apresentaram somente os analitos eluídos nos tempos de retenção dos mesmos.

Na Figura 4 é apresentado um cromatograma de água superficial analisada por headspace com CG-EM, onde podem ser visto os espectros de massa do benzeno e tolueno.

Para a escolha dos parâmetros a serem testados para avaliação da robustez dos métodos utilizaram-se os resultados do planejamento experimental multivariado. Tempo e fração de fase mostraram-se não significativos já no primeiro planejamento realizado durante a otimização do método de headspace, tanto com CG-DIC quanto com CG-EM. Portanto, os métodos são seguramente robustos em relação a estes fatores. Dessa forma, para determinação da robustez dos métodos foram avaliados os efeitos nas variações da resposta com relação à quantidade de cloreto de sódio (2,00 e 2,50 g para HS e MEFS) e 


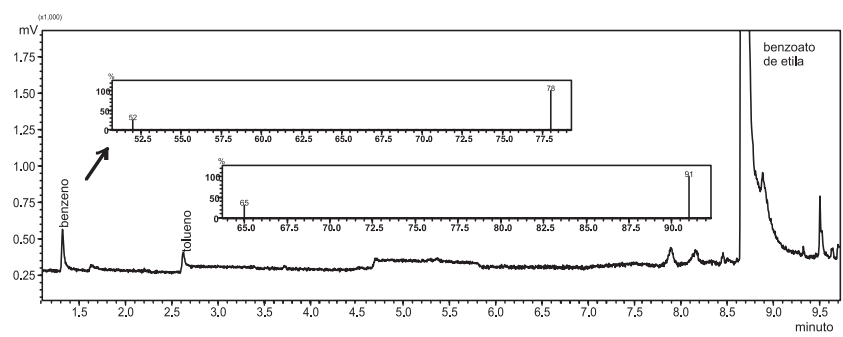

Figura 4. Cromatograma com os espectros de massas de dois componentes de uma amostra de água do Ribeirão Tripuí adicionada de benzoato de etila (PI) e extraída por HS e analisada por CG-EM

à temperatura $\left(70\right.$ e $80{ }^{\circ} \mathrm{C}$ para $\mathrm{HS}$ e 30 e $40{ }^{\circ} \mathrm{C}$ para a adsorção por MEFS). Os ensaios foram realizados mantendo-se os demais fatores constantes nos valores otimizados anteriormente. Os gráficos de pareto obtidos dessa avaliação mostraram que as estimativas dos efeitos para todos os analitos extraídos, tanto por headspace e analisados por CG-DIC e CG-EM quanto por MEFS e analisados por CG-EM, se encontram abaixo do limite delimitado por $p(0,05)$, como pode ser observado para o benzeno na Figura 5. Portanto, nenhum dos fatores avaliados dentro destes domínios modifica significativamente o sinal analítico gerado (a área dos picos cromatográficos) nesse nível de confiança, mostrando que os métodos são robustos.

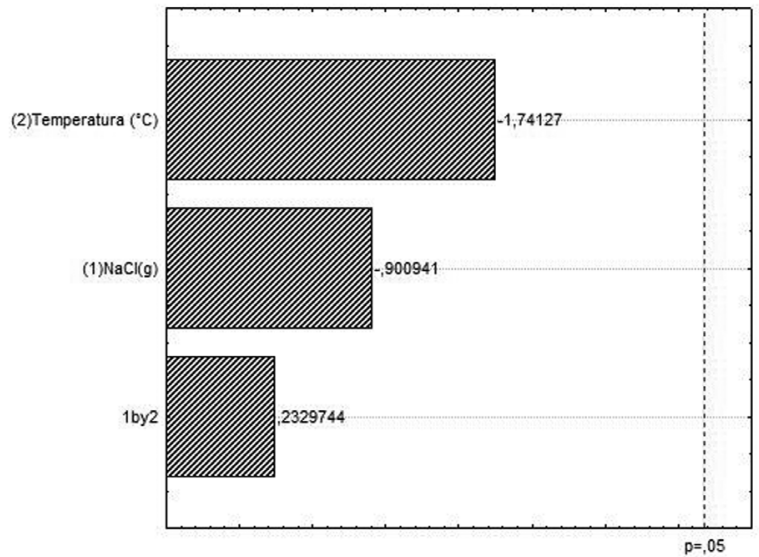

Figura 5. Diagrama de pareto para avaliação da variação da temperatura e da massa de cloreto de sódio na extração de benzeno de água por MEFS e analisado por CG-MS

Para a avaliação da linearidade dos métodos foram utilizadas soluções oriundas de uma amostra de água do Ribeirão Tripuí fortificada com seis concentrações diferentes de BTEX para o método de extração por HS e análise com CG-DIC e com sete para os métodos utilizando HS com CG-EM e MEFS com CG-EM. Cada uma das soluções das curvas analíticas obtidas foi analisada em sete replicatas no mesmo dia e, pelos resultados obtidos com a solução fortificada com $100 \mu \mathrm{g} \mathrm{L}^{-1}$ de BTEX, determinou-se a precisão do método com detecção por DIC e a precisão do método com detecção por EM foi determinada com a solução fortificada com $50 \mu \mathrm{g} \mathrm{L}^{-1}$ de BTEX.

O limite de detecção foi considerado como a concentração correspondente a três vezes o valor da área média do ruído dos cromatogramas das soluções mais diluídas e para o limite de quantificação considerouse a área equivalente a dez vezes aquela do ruído. Alguns resultados de validação dos métodos estudados, como a linearidade obtida dos parâmetros da curva analítica, os limites de detecção e quantificação relacionados à detectabilidade, à precisão e às recuperações dos analitos mostrando a exatidão, estão apresentados na Tabela 3.
Os resultados obtidos demonstram que a linearidade dos três métodos é muito boa para todos os compostos avaliados, pois não foi encontrado valor de $\mathrm{r}^{2}$ inferior a 0,99 . Os melhores resultados de precisão foram obtidos com o método de extração por MEFS e análise com CG-EM, pois nenhum composto apresentou variância maior que 5\%, enquanto que o método de extração por HS e análise com CG-EM apresentou valores de variância acima de $20 \%$ para todos os compostos avaliados.

As recuperações dos três métodos foram determinadas através da análise de nove replicatas de amostras fortificadas de BTEX em três concentrações diferentes e os resultados encontrados foram bastante satisfatórios. Com exceção dos isômeros $m$, $p$-xilenos extraídos por HS e analisados com CG-EM, que apresentaram pior resultado de recuperação, todos os demais resultados encontrados estiveram entre 80 e $110 \%$. Os menores limites de detecção de BTEX foram obtidos nos extratos de HS analisados com CG-EM, sendo que esses resultados variaram entre 0,002 e $0,007 \mu \mathrm{g} \mathrm{L}^{-1}$. Apesar desse método ter sido muito sensível para a detecção dos BTEX, não foi possível obter com ele os melhores resultados de variância e recuperação.

Os limites de detecção para todos os compostos avaliados com esse método foram inferiores aos encontrados por outros autores empregando a mesma técnica. ${ }^{1,7,8}$ Com o método de extração por HS e análise com CG-DIC as concentrações limites de BTEX detectadas estiveram entre 0,088 e $0,702 \mu \mathrm{g} \mathrm{L}{ }^{-1}$, sendo que para alguns dos BTEX avaliados esses resultados são inferiores aos obtidos em outras pesquisas semelhantes utilizando a mesma técnica analítica. ${ }^{4,5,33}$ A análise dos extratos de MEFS com CG-EM, além de apresentar os melhores resultados de precisão e exatidão, também apresentou baixos limites de detecção para os BTEX, entre 0,024 e $0,310 \mu \mathrm{g} \mathrm{L}^{-1}$. Essas concentrações são inferiores às encontradas por Wang et al. com relação a todos os BTEX. ${ }^{8}$ Concentrações abaixo de $0,5 \mathrm{ng} \mathrm{L}^{-1}$ para todos os compostos avaliados foram obtidas por Lee et al. ${ }^{22}$ porém, esses pesquisadores empregaram a técnica de pré-concentração por criogenia acoplada à extração por MEFS na fase HS e análise com CG-EM.

\section{BTEX em água em Ouro Preto, MG}

A presença de BTEX em amostras de água de abastecimento da Universidade Federal de Ouro Preto (UFOP) e do Ribeirão Tripuí, que passa pela região urbana de Ouro Preto, foi determinada por MEFS na fase HS e análise com CG-EM. Esse método analítico foi escolhido porque com ele foram obtidos os melhores resultados para os parâmetros de validação (Tabela 3).

Para a quantificação de BTEX nas amostras coletadas em triplicata utilizou-se benzoato de etila como padrão interno. O benzeno não foi encontrado em qualquer amostra analisada. $\mathrm{O}$ tolueno foi encontrado em algumas amostras, porém, em apenas uma amostra de um dos poços artesianos a concentração encontrada foi quantificável (329 ng L $\left.\mathrm{L}^{-1}\right)$. As demais amostras onde se constatou tolueno foram coletadas nos reservatórios de água. $\mathrm{Na}$ amostra de água do mesmo poço artesiano onde se quantificou tolueno também foram encontrados os isômeros meta- e/ou para-xilenos em concentrações quantificáveis $\left(283 \mathrm{ng} \mathrm{L}^{-1}\right)$. $\mathrm{O}$ isômero $o$-xileno encontrado nesse poço não foi quantificável. Esses resultados indicam a necessidade de uma avaliação mais profunda das águas desse poço artesiano porque, embora tenha sido perfurado a menos de 3 anos e ainda não esteja fornecendo água para o abastecimento humano, está situado numa região relativamente próxima a um local onde funcionou um posto de abastecimento de combustível até cerca de 3 anos atrás. Os isômeros de xileno também foram encontrados nas amostras coletadas no Ribeirão Tripuí e em um dos reservatórios de água da UFOP, no entanto, apenas nesse último a concentração de meta- e/ou para-xilenos foi quantificável $\left(0,234 \mu \mathrm{g} \mathrm{L}^{-1}\right)$. 
Tabela 3. Valores para os parâmetros de validação dos métodos de extração avaliados: linearidade (A, B e r²), detectabilidade (LD e LQ), precisão e recuperação (exatidão)

\begin{tabular}{|c|c|c|c|c|c|}
\hline & \multicolumn{5}{|c|}{ Método por HS com CG-DIC } \\
\hline & Benzeno & Tolueno & Etilbenzeno & $m, p$-xilenos & $o$-xileno \\
\hline Coeficiente angular (A) & 0,0074 & 0,0081 & 0,0094 & 0,0087 & 0,0125 \\
\hline Coeficiente linear (B) & 0,0074 & 0,0073 & 0,0327 & 0,0084 & 0,0138 \\
\hline$r^{2}$ & 0,9995 & 0,9992 & 0,9985 & 0,9980 & 0,9940 \\
\hline $\mathrm{LD}\left(\mu \mathrm{g} \mathrm{L}^{-1}\right)$ & 0,088 & 0,092 & 0,702 & 0,617 & 0,542 \\
\hline $\mathrm{LQ}\left(\mu \mathrm{g} \mathrm{L}^{-1}\right)$ & 0,293 & 0,306 & 2,340 & 2,057 & 1,807 \\
\hline Precisão (\%) & 13,39 & 10,82 & 12,26 & 8,89 & 10,87 \\
\hline \multirow[t]{3}{*}{ Exatidão (\%) } & 101,49 & 104,74 & 92,35 & 107,67 & 85,94 \\
\hline & \multicolumn{5}{|c|}{ Método por HS com CG-EM } \\
\hline & Benzeno & Tolueno & Etilbenzeno & $m, p$-xilenos & $o$-xileno \\
\hline Coeficiente angular & 0,0072 & 0,0075 & 0,0071 & 0,0068 & 0,0079 \\
\hline Coeficiente linear & 0,0023 & 0,0072 & 0,0013 & 0,0041 & 0,0005 \\
\hline$r^{2}$ & 0,9978 & 0,9961 & 0,9967 & 0,9973 & 0,9977 \\
\hline $\mathrm{LD}\left(\mu \mathrm{g} \mathrm{L}^{-1}\right)$ & 0,0023 & 0,0042 & 0,0027 & 0,0061 & 0,0071 \\
\hline $\mathrm{LQ}\left(\mu \mathrm{g} \mathrm{L}^{-1}\right)$ & 0,0078 & 0,0140 & 0,0089 & 0,0203 & 0,0235 \\
\hline Precisão (\%) & 28,77 & 22,50 & 21,74 & 22,34 & 20,74 \\
\hline \multirow[t]{3}{*}{ Exatidão (\%) } & 106,90 & 97,58 & 81,36 & 59,38 & 107,51 \\
\hline & \multicolumn{5}{|c|}{ Método por HS e MEFS com CG-EM } \\
\hline & Benzeno & Tolueno & Etilbenzeno & $m, p$-xilenos & $o$-xileno \\
\hline Coeficiente angular & 0,1699 & 0,1091 & 0,0852 & 0,0924 & 0,0804 \\
\hline Coeficiente linear & 0,0666 & 0,0858 & 0,0055 & 0,0943 & 0,0069 \\
\hline$r^{2}$ & 0,9992 & 0,9990 & 0,9995 & 0,9991 & 0,9993 \\
\hline $\mathrm{LD}\left(\mu \mathrm{g} \mathrm{L}^{-1}\right)$ & 0,097 & 0,089 & 0,310 & 0,052 & 0,024 \\
\hline $\mathrm{LQ}\left(\mu \mathrm{g} \mathrm{L^{-1 }}\right)$ & 0,325 & 0,297 & 1,032 & 0,173 & 0,082 \\
\hline Precisão (\%) & 4,70 & 3,77 & 2,82 & 2,28 & 4,33 \\
\hline Exatidão (\%) & 104,30 & 97,95 & 99,54 & 102,76 & 102,60 \\
\hline
\end{tabular}

LD = Limite de Detecção, LD = Limite de Quantificação e $r^{2}=$ coeficiente de correlação linear

Vale ressaltar que na única amostra onde foi possível se quantificar tolueno o valor encontrado foi mais de 500 vezes inferior à concentração limite legal para potabilidade da água. ${ }^{2}$ A legislação estipula um limite para a soma das concentrações dos isômeros orto-, meta- e para-xilenos igual a $300 \mathrm{mg} \mathrm{L}^{-1}$ em água potável, ${ }^{2}$ porém, as concentrações encontradas foram cerca de mil vezes menores.

É importante realçar que os resultados de concentrações de BTEX encontrados neste trabalho são importantes para despertar outras avaliações mais amplas, porque a água do poço artesiano e dos três reservatórios na UFOP é consumida sem qualquer pré-tratamento.

Tabela 4. Concentração de BTEX nas amostras de águas coletadas na superfície do Ribeirão Tripuí (S) em Ouro Preto, nos poços de abastecimento (P) e reservatórios (R) da UFOP, amostrados conforme descrito na Parte Experimental

\begin{tabular}{lccccc}
\hline \multirow{2}{*}{ Amostra } & \multicolumn{5}{c}{ Concentração $\left(\mu \mathrm{L} \mathrm{L}^{-1}\right)$} \\
& Benzeno & Tolueno & Etilbenzeno & $m, p$-xilenos & $o$-xileno \\
\hline S1 & $<\mathrm{LD}$ & $<\mathrm{LD}$ & $<\mathrm{LD}$ & $0,131^{*}$ & $0,039^{*}$ \\
S2 & $<\mathrm{LD}$ & $<\mathrm{LD}$ & $<\mathrm{LD}$ & $0,054^{*}$ & $0,028^{*}$ \\
S3 & $<\mathrm{LD}$ & $<\mathrm{LD}$ & $<\mathrm{LD}$ & $<\mathrm{LD}$ & $0,025^{*}$ \\
P1 & $<\mathrm{LD}$ & 0,329 & $<\mathrm{LD}$ & 0,283 & $0,046^{*}$ \\
P2 & $<\mathrm{LD}$ & $<\mathrm{LD}$ & $<\mathrm{LD}$ & $<\mathrm{LD}$ & $<\mathrm{LD}$ \\
R1 & $<\mathrm{LD}$ & $0,147^{*}$ & $<\mathrm{LD}$ & $<\mathrm{LD}$ & $<\mathrm{LD}$ \\
R2 & $<\mathrm{LD}$ & $0,178^{*}$ & $<\mathrm{LD}$ & $<\mathrm{LD}$ & $<\mathrm{LD}$ \\
R3 & $<\mathrm{LD}$ & $0,242^{*}$ & $<\mathrm{LD}$ & 0,234 & $0,038^{*}$ \\
\hline
\end{tabular}

* Concentração inferior ao LQ (Limite de Quantificação). LD = Limite de Detecção, conforme Tabela 3.

\section{CONCLUSÕES}

Para a avaliação de BTEX em amostras de água designada ao consumo humano é necessário o emprego de técnicas de pré-concentração, devido às baixas concentrações em que esses compostos são encontrados nessas matrizes.

O emprego de planejamento experimental multivariado para otimização de condições analíticas para a determinação de BTEX em água utilizando cromatografia gasosa mostrou-se bastante adequado, devido ao grande número de variáveis analíticas envolvidas nos métodos avaliados. O método de extração por MEFS no HS e análise com CG-EM mostrou ser linear $\left(\mathrm{r}^{2}>0,999\right)$, preciso (variância menor que 5\%), exato (recuperação entre 98 e 104\%) e sensível (LD entre 24 e $310 \mathrm{ng} \mathrm{L}^{-1}$ ), apresentando os melhores resultados para os parâmetros de validação. No entanto, o método de extração por HS e análise com CG-EM, embora tenha apresentado os piores resultados de precisão (variância entre 20 e 30\%), mostrou ser extremamente sensível para a análise de BTEX em água com valores de LD entre 2 e $7 \mathrm{ng} \mathrm{L}^{-1}$. Vale destacar a utilidade do método de extração por HS e análise com CG-DIC para avaliação de potabilidade da água com a relação a BTEX. Os limites de detecção de BTEX em água por esse método (LD entre 88 e 702 $n \mathrm{~L} \mathrm{~L}^{-1}$ ) são cerca de 50 vezes inferiores à concentração máxima permitida em água potável para o benzeno e até 1850 vezes para o tolueno. ${ }^{2}$ Essa técnica merece atenção pelo fato de ser bastante simples e de baixo custo, necessitando apenas de frascos de headspace, um forno com temperatura estável, podendo ser utilizado o próprio forno do cromatógrafo, e um equipamento de CG-DIC, disponível em muitos laboratórios analíticos. 


\section{REFERÊNCIAS}

1. Nogueira, D. A.; Dissertação de Mestrado, Universidade Federal de Ouro Preto, Brasil, 2006.

2. Brasil; Portaria ${ }^{\circ}$ 518, Ministério da Saúde: Brasília, 2004.

3. Zwank, L.; Schmidt, T. C.; Haderlein, S. B.; Berg, M.; Environ. Sci. Technol. 2002, 36, 2054.

4. Lacorte, S.; Olivella, L.; Rosell, M.; Figueras, M.; Ginebreda, A.; Barceló, D.; Chromatographia 2002, 56, 739.

5. Menéndez, J. C. F.; Sánchez, M. L. F.; Uria, J. E. S.; Martinez, E. F.; Sanz-Medel, A.; Anal. Chim. Acta 2000, 415, 9.

6. Pavon, J. L. P.; Sanchez, M. D. N.; Laespada, M. E. F.; Cordero, B. M.; J. Chromatogr., A 2007, 1175, 106.

7. Serrano, A.; Gallego, M.; J. Chromatogr., A 2004, 1045, 181.

8. Wang, Z.; Li, K.; Fingas, M.; Sigouin, L.; Menard, L.; J. Chromatogr., A 2002, 971, 173.

9. Bianchi, F.; Careri, M.; Marengo, E.; Musci, M.; J. Chromatogr., A 2002, 975, 113 .

10. Dórea, H. S.; Bispo, J. R. L.; Aragão, K. A. S.; Cunha, B. B.; Navickiene, S.; Alves, J. P. H.; Romão, L. P. C.; Garcia, C. A. B.; Microchem. J. 2007, 85, 234.

11. Kubinec, R.; Berezkin, V.; Górová, R.; Addová, G.; Mracnová, H.; Soják, L.; J. Chromatogr., B 2004, 800, 295.

12. Rego, E. C. P. D.; Netto, A. D. P.; Bull. Environ. Contam. Toxicol. 2007, 79, 660 .

13. Rosell, M.; Lacorte, S.; Ginebreda, A.; Barcelo, D.; J. Chromatogr., A 2003, 995, 171.

14. Popp, P.; Paschke, A.; Chromatographia 1997, 46, 419.

15. Cho, H.-J.; Baek, K.; Lee, H.-H.; Lee, S.-H.; Yang, J.-W.; J. Chromatogr., A 2003, 988, 177.

16. Djozan, D.; Assadi, Y.; Chromatographia 1997, 45, 183.

17. Djozan, D.; Pournaghi-Azar, M. H.; Bahar, S.; Chromatographia 2004, $59,595$.
18. Farajzadeh, M.; Matin, A.; Chromatographia 2008, 68, 443.

19. Gaujac, A.; Emídio, E. S.; Navickiene, S.; Ferreira, S. L. C.; Dórea, H. S.; J. Chromatogr., A 2008, 1203, 99.

20. Ji, J.; Deng, C.; Shen, W.; Zhang, X.; Talanta 2006, 69, 894.

21. Jochmann, M.; Yuan, X.; Schmidt, T.; Anal. Bioanal. Chem. 2007, 387 , 2163.

22. Lee, M.-R.; Chang, C.-M.; Dou, J.; Chemosphere 2007, 69, 1381.

23. Sieg, K.; Fries, E.; Puttmann, W.; J. Chromatogr., A 2008, 1178, 178.

24. Aeppli, C.; Berg, M.; Hofstetter, T. B.; Kipfer, R.; Schwarzenbach, R. P.; J. Chromatogr., A 2008, 1181, 116.

25. Sarafraz-Yazdi, A.; Amiri, A. H.; Es 'haghi, Z.; Chemosphere 2008, 71, 671.

26. Mohammadi, A.; Alizadeh, N.; J. Chromatogr., A 2006, 1107, 19.

27. Jurdáková, H.; Kubinec, R.; Jurcisinová, M.; Krkosová, Z.; Blasko, J.; Ostrovský, I.; Soják, L.; Berezkin, V. G.; J. Chromatogr., A 2008, 1194, 161.

28. Ferreira, F. C. S.; Tese de Doutorado, Universidade de São Paulo, Brasil, 2006.

29. Pawliszyn, J.; Solid-Phase Microextraction: Theory and Practice, Willey-VHC: New York, 1997.

30. Barros Neto, B.; Scarminio, I. S.; Bruns, R. E.; Como Fazer Experimentos: pesquisa e desenvolvimento na ciência e na indústria, Ed. da Unicamp: Campinas, 2003.

31. INMETRO - Instituto Nacional de Metrologia Normalização e Qualidade Industrial; Orientações sobre Validação de Métodos de Ensaios Químicos, Documento Orientativo: DOQ-CGCRE-008, 2003.

32. Ribani, M.; Bottoli, C. B. G.; Collins, C. H.; Jardim, I. C. S. F.; Melo, L. F. C.; Quim. Nova 2004, 27, 771.

33. Alonso, A.; Fernández-Torroba, M. A.; Tena, M. T.; Pons, B.; Chromatographia 2003, 57, 369. 